\title{
Fault Tolerant Control for Uncertain Time-Delay Systems with a Trajectory Tracking Approach
}

\author{
ShiLei Zhao, Hong Guo, and YuPeng Liu \\ School of SoftWare, Harbin University of Science and Technology, Harbin 150036, China \\ Correspondence should be addressed to ShiLei Zhao; zhaosl1210@126.com
}

Received 13 September 2014; Revised 31 December 2014; Accepted 2 January 2015

Academic Editor: Vincent Cocquempot

Copyright (C) 2015 ShiLei Zhao et al. This is an open access article distributed under the Creative Commons Attribution License, which permits unrestricted use, distribution, and reproduction in any medium, provided the original work is properly cited.

\begin{abstract}
This paper studies the problem of fault tolerant control by trajectory tracking for a class of linear constant time-delay systems. The aim is to design a control law by considering the fault detected by the observer to make the faulty system track the reference model even if faults occur. By considering two kinds of actuator faults, one constant and another time-varying, the corresponding proportional integral observers and active FTC control laws are designed, respectively. State tracking error, state estimation error, output estimation error, and fault estimation error are combined into a descriptor system. Based on Lyapunov-Krasovskii functional approach stability problems of the descriptor system are easily solved in terms of the Linear Matrix Inequalities (LMI). Finally, a numerical example is considered to prove the effectiveness in both cases.
\end{abstract}

\section{Introduction}

Over the past few decades, problems of fault tolerant control, well known as FTC, in dynamic systems have attracted lots of attention $[1,2]$. FTC has been developed to preserve the system stability and maintain acceptable performances in case of faults occurring. The existing FTC strategies can be divided into two categories. The first one, named as the passive FTC, treats the fault as uncertainty; therefore, it involves no fault detection and estimation (see [3-5]). The second one, the active FTC, differs from the passive FTC in that it requires a fault detection and isolation (FDI) block to detect, isolate, and estimate faults which are used to compensate the fault and ensure an acceptable system performance (e.g., [6-8]). As the obtained fault information is used, the active FTC is more reliable.

On the other hand, time-delay is another factor that can degrade system performance; it is a built-in feature in many engineering systems. The presence of time-delay, together with faults, could cause system to be instable easily. Therefore, researching on FTC design of time-delay system has great practical and theoretical significance [9]; this challenging topic has ignited the interest of some authors. For example, $[10,11]$ provide a kind of fault detection method based on an iterative learning observer for nonlinear constant state delay systems. Reference [12] designs $H_{\infty}$ fault detection filters for multiple time-delay discrete-time systems. Based on a switched descriptor observer approach, [13] deals with sensor fault estimation and compensation problems of time-delay switched systems. In [14], for both additive and multiplicative faults, a robust fault detection and isolation scheme is proposed for uncertain continuous linear systems with discrete state delays. In [15], a fault detection filter is investigated for a class of discrete-time switched linear systems with timevarying delays so that the different estimation errors are minimized. In [16], some adaptive fault diagnosis observers (AFDO) are designed to deal with fast fault estimation and accommodation problems for time-varying delay systems.

Recently, there is also an active FTC approach based on trajectory tracking, developed to solve the FTC problem. This scheme is composed of faulty system, reference model, observer, and controller and its aim is to design a control law by considering actuator faults detected by observers and to make the faulty system states track the reference model states which are not effected by faults [17-19].

This paper is about to develop a strategy for linear constant time-delay systems based on trajectory tracking. The motivation of this paper mainly stems from two facts: 
(1) some FTC schemes of time-delay systems are obtained $[20,21]$, but less work which studies on FTC problems employs the descriptor redundancy property and solves the fault isolation, estimation, and FTC problems together; (2) there is some work addressing FTC designs based on trajectory tracking which focused on linear time invariant (LTI) system without time-delay, but few work is focused on FTC of time-delay systems. Our work will extend earlier results of fault estimation using trajectory tracking to the time-delay systems.

In this paper, our purpose is to study the FTC design problem for linear state time-delay systems subjected to constant or time-varying faults. The main idea is to design an active FTC controller and PI observer and to use the virtual dynamic [22-24] in both active FTC law and output estimation error expression to turn the problem under study into a descriptor system. By using the Lyapunov-Krasovskii functional approach, the stability of the descriptor system has been proved. The advantages of the proposed method is also based on the above two facts: (1) the introduction of trajectory tracking can ensure the tracking of faulty systems to reference models, which could guarantee an acceptable system performance even if faults occur; (2) the descriptor redundancy property can avoid crossed terms in the LMI and then decrease the number of LMI conditions and consequently relax the conservatism [17].

This paper is organized as follows. In the next section, the system under study and the active FTC scheme based on trajectory tracking are presented. In Section 3, FTC design for linear state time-delay systems affected by constant fault without uncertainties is established. Then, some FTC design for linear state time-delay systems affected by time-varying faults with uncertainties is given. In the last section, a numerical example for constant faults without uncertainties and time-varying faults with uncertainties is considered to illustrate the applicability and effectiveness of the proposed approaches.

Notations. In a block matrix, the notation * stands for the terms induced by symmetry. The superscript $T$ denotes matrix transpose, $\operatorname{sym}(A)$ denotes $A+A^{T}$, and $\operatorname{diag}\{\cdots\}$ stands for a block-diagonal matrix.

The following lemma is needed to provide LMI conditions.

Lemma 1. For any matrices $X, Y$, and $\Sigma(t)$ with appropriate dimensions and $\Sigma^{T}(t) \Sigma(t)<I$ and for any positive real number $t$, it follows that

$$
X^{T} \Sigma^{T}(t) Y+Y^{T} \Sigma(t) X \leq t X^{T} X+t^{-1} Y^{T} Y .
$$

\section{Problem Formulation}

Consider the following system without faults corresponding to a reference model:

$$
\begin{gathered}
\dot{x}(t)=A x(t)+B u(t)+A_{d} x(t-h), \\
y=C x(t)+C_{d} x(t-h),
\end{gathered}
$$

where $x(t) \in R^{n}$ is the state vector, $u(t) \in R^{m}$ is the input vector, and $y(t) \in R^{p}$ is the output vector. $A, B, A_{d}, C$, and $C_{d}$ are known constant real matrices of appropriate dimensions. $h$ is the state delay and $h \in R^{+}$is a constant real number.

Consider the faulty system given by

$$
\begin{aligned}
& \dot{x}_{f}(t)=\bar{A} x_{f}(t)+\bar{A}_{d} x_{f}(t-h)+\bar{B} u_{f}(t)+\bar{B} f(t), \\
& y_{f}(t)=\bar{C} x_{f}(t)+\bar{C}_{d} x_{f}(t-h),
\end{aligned}
$$

where $x_{f}(t) \in R^{n}, u_{f}(t) \in R^{m}, y_{f}(t) \in R^{p}$, and $f(t) \in R^{m}$ are the faulty state vector, the fault tolerant control vector, the faulty output vector, and the fault vector affecting the system behavior. And the uncertainties of system (3) are defined by

$$
\bar{X}(t)=X+\Delta X(t), \quad X \in\left\{A, A_{d}, B, C, C_{d}\right\},
$$

where $\Delta A, \Delta A_{d}, \Delta B, \Delta C$, and $\Delta C_{d}$ are time-varying unknown matrices describing the bounded model uncertainties, defined by

$$
\begin{gathered}
\Delta X(t)=M_{x} F(t) N_{x}, \\
(X, x)=\left\{(A, a),\left(A_{d}, a d\right),(B, b),(C, c),\left(C_{d}, c d\right)\right\},
\end{gathered}
$$

where $M_{a}, M_{a d}, M_{b}, M_{c}, M_{c d}, N_{a}, N_{a d}, N_{b}, N_{c}$, and $N_{c d}$ are known constant real matrices with appropriate dimensions and the matrix function $F(t)$ is bounded by

$$
F(t) F(t)^{T} \leq I
$$

In order to estimate the fault vector $f(t)$ which is required by the FTC scheme and the faulty system states $x_{f}(t)$, we consider the PI observer as follows:

$$
\begin{aligned}
& \dot{\hat{x}}_{f}(t)= A \widehat{x}_{f}(t)+A_{d} \widehat{x}_{f}(t-h)+B u_{f}(t) \\
&+B \widehat{f}(t)+H_{1}\left(y_{f}(t)-\widehat{y}_{f}(t)\right), \\
& \widehat{f}(t)= H_{2}\left(y_{f}(t)-\widehat{y}_{f}(t)\right), \\
& \hat{y}_{f}(t)=C \widehat{x}_{f}(t)+C_{d} \widehat{x}_{f}(t-h),
\end{aligned}
$$

where $H_{1} \in R^{n \times p}$ and $H_{2} \in R^{r \times p}$ are the observer's gain matrices to be determined.

\section{Fault Tolerant Controller Design}

In this section, two cases are considered according to the characteristics of faults. First, we assume that the fault is a constant one and there are no uncertainties in faulty system (6). Second, assume that the fault is a time-varying one and there are uncertainties in the faulty system.

The FTC design scheme is illustrated in Figure 1. The objective of this work is to ensure the tracking of the faulty system to the nominal one. In other words, the scheme is to design FTC law and observer gain matrices to minimize the differences between the faulty states of (3) and the reference states given by model (2), the faulty system states and the observer states, the faulty system estimation output and 


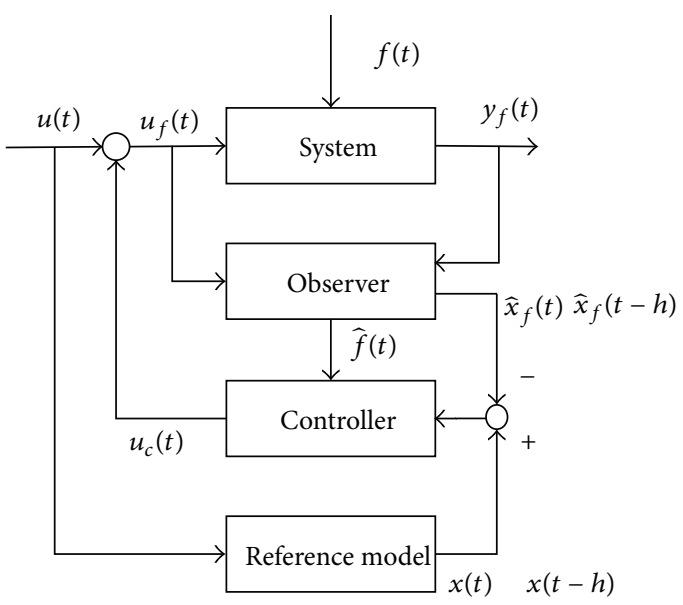

FIGURE 1: The controller scheme.

the reference model output, and the nominal input and the FTC input plus the fault.

From the FTC scheme of Figure 1, the following FTC law structure is proposed [17]:

$$
\begin{aligned}
u_{f}(t)= & u(t)+K_{1}\left(x(t)-\widehat{x}_{f}(t)\right)-\widehat{f}(t) \\
& +K_{2}\left(x(t-h)-\widehat{x}_{f}(t-h)\right),
\end{aligned}
$$

where $K_{1}, K_{2} \in R^{m \times n}$ are the state feedback gain matrices to guarantee the stability of the faulty system even if the fault occurs and minimize the difference between the faulty system and the reference one.

3.1. First Case: Constant Fault without Uncertainties. It is here considered that the fault which affects the system actuator is a constant bias. Obviously, it is a special case that the fault satisfies

$$
\dot{f}(t)=0 .
$$

In the following part, to ensure the tracking, we first give state error, fault estimation error, output error and tracking error, and the difference between nominal input and FTC input plus the fault, respectively, by

$$
\begin{aligned}
& e_{p}(t)=x(t)-x_{f}(t), \\
& e_{s}(t)=x_{f}(t)-\widehat{x}_{f}(t), \\
& e_{f}(t)=f(t)-\widehat{f}(t), \\
& e_{y}(t)=y_{f}(t)-\hat{y}_{f}(t), \\
& e_{u}(t)=u(t)-\left(u_{f}(t)+f(t)\right) .
\end{aligned}
$$

By using formulae (2), (3), (7), and (10), the dynamics of $e_{p}(t)$ and $e_{s}(t)$ are given by

$$
\begin{aligned}
& \dot{e}_{p}(t)=A e_{p}(t)+B e_{u}(t)+A_{d} e_{p}(t-h), \\
& \dot{e}_{s}(t)=A e_{s}(t)+B e_{f}(t)-H_{1} e_{y}(t)+A_{d} e_{s}(t-h) .
\end{aligned}
$$

The fault estimation error dynamics $e_{f}(t)$ is expressed as follows:

$$
\dot{e}_{f}(t)=\dot{f}(t)-\dot{\hat{f}}(t)=-H_{2} e_{y}(t) .
$$

The output estimation error $e_{y}(t)$ can be written in the form of

$$
e_{y}(t)=C e_{s}(t)+C_{d} e_{s}(t-h) .
$$

In order to organize the above equations into the form of descriptor systems, we can introduce a "virtual dynamics" in the output estimation error; this latter can be rewritten as

$$
0 \dot{e}_{y}(t)=C e_{s}(t)+C_{d} e_{s}(t-h)-e_{y}(t) .
$$

By adding and subtracting $K_{1} x_{f}(t), K_{2} x_{f}(t-h)$, and $f(t)$ in (8) and using (10), one can obtain

$$
\begin{aligned}
0 \dot{e}_{u}(t)= & K_{1} e_{p}(t)+K_{1} e_{s}(t)+K_{2} e_{p}(t-h) \\
& +K_{2} e_{s}(t-h)+e_{f}(t)+e_{u}(t) .
\end{aligned}
$$

The combinations of (11), (12), (14), and (15) yield a descriptor system expressed as follows:

$$
E \dot{e}(t)=\widetilde{A} e(t)+\widetilde{A}_{d} e(t-h),
$$

where $E=\operatorname{diag}\left\{I_{2 n+m}, 0_{m+p}\right\}$,

$$
\begin{gathered}
e(t)=\left(e_{p}^{T}(t), e_{s}^{T}(t), e_{f}^{T}(t), e_{u}^{T}(t), e_{y}^{T}(t)\right)^{T}, \\
\widetilde{A}=\left[\begin{array}{ccccc}
A & 0 & 0 & B & 0 \\
0 & A & B & 0 & -H_{1} \\
0 & 0 & 0 & 0 & -H_{2} \\
K_{1} & K_{1} & I & I & 0 \\
0 & C & 0 & 0 & -I
\end{array}\right], \\
\widetilde{A}_{d}=\left[\begin{array}{ccccc}
A_{d} & 0 & 0 & 0 & 0 \\
0 & A_{d} & 0 & 0 & 0 \\
0 & 0 & 0 & 0 & 0 \\
K_{2} & K_{2} & 0 & 0 & 0 \\
0 & C_{d} & 0 & 0 & 0
\end{array}\right]
\end{gathered}
$$

The main proposed result can now be established [25].

Theorem 2. The tracking error $e_{p}(t)$, the state error $e_{s}(t)$, and fault estimation error $e_{f}(t)$ asymptotically converge to zero if there exist some matrices $P_{1}>0, P_{2}>0, P_{3}>0$, and matrices $Y_{2}$ and $Y$ with appropriate dimensions, such that the following inequalities hold:

$$
\left[\begin{array}{cc}
\Omega & Y \\
Y^{T} & -\frac{P_{3}}{h}
\end{array}\right]<0,
$$


where the expression of $\Omega$ is shown as follows:

$$
\begin{aligned}
& \Omega=W_{p} P_{2} W_{p}^{T}+h W_{p 1} E^{T} P_{3} E W_{p 1}^{T}-W_{p h} P_{2} W_{p h}^{T} \\
& +\operatorname{sym}\left[W_{p} P_{1} W_{p 1}^{T}+Y E W_{P}^{T}+Y E W_{p h}^{T}\right. \\
& +\left(Y_{2} A_{0}-H C_{1}-X C_{2}\right) W_{p}^{T} \\
& \left.+\left(Y_{2} A_{d 0}-K C_{3}\right) W_{p h}^{T}\right], \\
& W_{p}=\left[\begin{array}{lll}
I_{2 n+2 m+p} & 0 & 0
\end{array}\right], \quad W_{p h}=\left[\begin{array}{lll}
0 & I_{2 n+2 m+p} & 0
\end{array}\right], \\
& W_{p 1}=\left[\begin{array}{lll}
0 & 0 & I_{2 n+2 m+p}
\end{array}\right], \\
& Y_{2}=\left[\begin{array}{lll}
\widetilde{Y}_{2}^{T} & \widetilde{Y}_{2}^{T} & \widetilde{Y}_{2}^{T}
\end{array}\right]^{T}, \quad X=\left[\begin{array}{lll}
\widetilde{X}^{T} & \widetilde{X}^{T} & \widetilde{X}^{T}
\end{array}\right]^{T}, \\
& \widetilde{X}=\left[\begin{array}{lllll}
0 & 0 & 0 & X_{41}^{T} & 0
\end{array}\right]^{T}, \quad K=\left[\begin{array}{lll}
\widetilde{K}^{T} & \widetilde{K}^{T} & \widetilde{K}^{T}
\end{array}\right]^{T} \text {, } \\
& \widetilde{K}=\left[\begin{array}{lllll}
0 & 0 & 0 & K_{42}^{T} & 0
\end{array}\right]^{T}, \quad H=\left[\begin{array}{llll}
\widetilde{H}^{T} & \widetilde{H}^{T} & \widetilde{H}^{T}
\end{array}\right]^{T}, \\
& \widetilde{H}=\left[\begin{array}{lllll}
0 & H_{21}^{T} & H_{32}^{T} & 0 & 0
\end{array}\right]^{T}, \quad C_{1}=\left[\begin{array}{lllll}
0 & 0 & 0 & 0 & I
\end{array}\right], \\
& C_{2}=\left[\begin{array}{lllll}
I & I & 0 & 0 & 0
\end{array}\right], \quad C_{3}=C_{2}, \\
& A_{0}=\left[\begin{array}{ccccc}
A & 0 & 0 & B & 0 \\
0 & A & B & 0 & 0 \\
0 & 0 & 0 & 0 & 0 \\
0 & 0 & I & I & 0 \\
0 & C & 0 & 0 & -I
\end{array}\right], \quad A_{d 0}=\left[\begin{array}{ccccc}
A_{d} & 0 & 0 & 0 & 0 \\
0 & A_{d} & 0 & 0 & 0 \\
0 & 0 & 0 & 0 & 0 \\
0 & 0 & 0 & 0 & 0 \\
0 & C_{d} & 0 & 0 & 0
\end{array}\right], \\
& \tilde{Y}_{2}=\left[\begin{array}{ccccc}
Y_{11} & 0 & 0 & 0 & 0 \\
0 & Y_{12} & 0 & 0 & 0 \\
0 & 0 & Y_{13} & 0 & 0 \\
Y_{16} & 0 & 0 & Y_{14} & Y_{18} \\
Y_{17} & 0 & 0 & 0 & Y_{15}
\end{array}\right]
\end{aligned}
$$

where $Y_{12}, Y_{13}$, and $Y_{14}$ are invertible matrices and $Y_{11}, Y_{15}$, $Y_{16}, Y_{17}$, and $Y_{18}$ are slack matrices.

The observer and controller gains are then computed by

$$
\begin{array}{ll}
K_{1}=\left(Y_{14}\right)^{-1} X_{41}, & K_{2}=\left(Y_{14}\right)^{-1} K_{42}, \\
H_{1}=\left(Y_{12}\right)^{-1} H_{21}, & H_{2}=\left(Y_{13}\right)^{-1} H_{32} .
\end{array}
$$

Remark 3. It should be mentioned that the free weighting matrix $Y_{2}$ has the structure of $Y_{2}=\left[\begin{array}{ccc}\widetilde{Y}_{2}^{T} & \widetilde{Y}_{2}^{T} & \widetilde{Y}_{2}^{T}\end{array}\right]^{T}$. But the conservatism would increase; in order to obtain a tractable matrix condition, we can adopt the method by defining

$$
Y_{2}=\left[\begin{array}{ccc}
\alpha_{1} I & 0 & 0 \\
0 & \alpha_{2} I & 0 \\
0 & 0 & \alpha_{3} I
\end{array}\right]\left[\begin{array}{l}
\widetilde{Y}_{2} \\
\widetilde{Y}_{2} \\
\widetilde{Y}_{2}
\end{array}\right],
$$

where $\alpha_{1}, \alpha_{2}$, and $\alpha_{3}$ are the real number.

By choosing these scalars appropriately, the conservatism cannot increase much. This method has been used by [23].
Proof. Choose a Lyapunov-Krasovskii functional candidate as

$$
\begin{aligned}
& V(t)=V_{1}(t)+V_{2}(t)+V_{3}(t), \\
& V_{1}(t)=e^{T}(t) E^{T} P_{1} e(t), \\
& V_{2}(t)=\int_{-h}^{0} \int_{t+v}^{t} \dot{e}^{T}(\omega) E^{T} P_{3} E \dot{e}(\omega) d \omega d v, \\
& V_{3}(t)=\int_{t-h}^{t} e^{T}(\omega) P_{2} e(\omega) d \omega,
\end{aligned}
$$

where $P_{1}$ satisfies $E^{T} P_{1}=P_{1} E$.

The time derivatives of $V(t)$ are given by

$$
\begin{aligned}
\dot{V}= & 2 e^{T}(t) P_{1} E \dot{e}(t)+h \dot{e}^{T}(t) E^{T} P_{3} E \dot{e}(t) \\
& -\int_{t-h}^{t} \dot{e}^{T}(\omega) E^{T} P_{3} E \dot{e}(\omega) d \omega+e^{T}(t) P_{2} e(t) \\
& -e^{T}(t-h) P_{2} e(t-h) .
\end{aligned}
$$

According to the Newton-Lebniz formula and closedloop system equation, the following equations are true:

$$
\begin{aligned}
& \Pi_{1}=\xi^{T}(t) Y E\left[e(t)-e(t-h)-\int_{t-h}^{t} \dot{e}(\omega) d \omega\right]=0, \\
& \Pi_{2}=\xi^{T}(t) Y_{2}\left[\widetilde{A} e(t)-\widetilde{A}_{d} e(t-h)-E \dot{e}(t)\right]=0,
\end{aligned}
$$

where $Y, Y_{2}$ are appropriate dimensioned matrices and $\xi(t)=$ $\left[e^{T}(t), e^{T}(t-h),(E \dot{e}(t))^{T}\right]^{T}$.

Adding $2 \Pi_{1}, 2 \Pi_{2}$, and $h \xi^{T}(t) Y P_{3}^{-1} Y^{T} \xi(t)$ and subtracting $\int_{t-h}^{t} \xi^{T}(t) Y P_{3}^{-1} Y^{T} \xi(t) d \omega$, respectively, in (24) yield (26) which is shown as follows:

$$
\begin{aligned}
\dot{V}(t)= & 2 e^{T}(t) E^{T} P_{1} \dot{e}(t)+h \dot{e}^{T}(t) E^{T} P_{3} E \dot{e}(t) \\
& -\int_{t-h}^{t} \dot{e}^{T}(\omega) E^{T} P_{3} E \dot{e}(\omega) d \omega+e^{T}(t) P_{2} e(t) \\
& -e^{T}(t-h) P_{2} e(t-h) \\
& +2 \xi^{T}(t) Y E\left[e(t)-e(t-h)-\int_{t-h}^{t} \dot{e}(\omega) d \omega\right] \\
& +2 \xi^{T}(t) Y_{2}\left[\widetilde{A} e(t)+\widetilde{A}_{d} e(t-h)-E \dot{e}(t)\right] \\
= & 2 e^{T}(t) P_{1} E \dot{e}^{T}(t)+h \dot{e}^{T}(t) E^{T} P_{3} E \dot{e}(t) \\
& -\int_{t-h}^{t} \dot{e}^{T}(\omega) E^{T} P_{3} E \dot{e}(\omega) d \omega+e^{T}(t) P_{2} e(t) \\
& -e^{T}(t-h) P_{2} e(t-h) \\
& +2 \xi^{T}(t) Y E\left[e(t)-e(t-h)-\int_{t-h}^{t} \dot{e}(\omega) d \omega\right] \\
& +2 \xi^{T}(t) Y_{2}\left[\widetilde{A} e(t)+\widetilde{A}_{d} e(t-h)-E \dot{e}(t)\right] \\
& +h \xi^{T}(t) Y P_{3}^{-1} Y^{T} \xi(t)-\int_{t-h}^{t} \xi^{T}(t) Y P_{3}^{-1} Y^{T} \xi(t) d \omega
\end{aligned}
$$




$$
\begin{aligned}
=\xi^{T}(t) & \Omega \xi(t)+h \xi^{T}(t) Y P_{3}^{-1} Y^{T} \xi(t) \\
-\int_{t-h}^{t} & {\left[\xi^{T}(t) Y+\dot{e}^{T}(\omega) E^{T} P_{3}\right] } \\
& \times P_{3}^{-1}\left[Y^{T} \xi(t)+P_{3} E \dot{e}(\omega)\right] d \omega .
\end{aligned}
$$

Then, we know from (26) that $\Omega+h Y P_{3}^{-1} Y^{T}<0$ which guarantees $\dot{V}(t)$ is nonpositiveness for nonzero $\xi(t)$. One can always find a sufficiently small $\epsilon>0$ such that $\dot{V}(t) \leq-\epsilon\|e(t)\|^{2}$ and the asymptotic stability of the system is proved.

3.2. Second Case: Time-Varying Fault with Uncertainties. In this part, we consider system (3) with the uncertainties and the fault being time-varying one. Here, we modify PI observer slightly as follows:

$$
\begin{gathered}
\dot{\hat{x}}_{f}(t)=A \widehat{x}_{f}(t)+A_{d} \widehat{x}_{f}(t-h)+B u_{f}(t) \\
+B \widehat{f}(t)+H_{1}\left(y_{f}(t)-\widehat{y}_{f}(t)\right), \\
\dot{\hat{f}}(t)=H_{2}\left(y_{f}(t)-\widehat{y}_{f}(t)\right)-\widehat{f}(t), \\
\widehat{y}_{f}(t)=C \widehat{x}_{f}(t)+C_{d} \widehat{x}_{f}(t-h) .
\end{gathered}
$$

The dynamics of $e_{p}(t), e_{s}(t)$, and $e_{f}(t)$ are given by

$$
\begin{aligned}
\dot{e}_{p}(t)= & A e_{p}(t)+\Delta A e_{p}(t)+B e_{u}(t)+\Delta B e_{u}(t) \\
& +A_{d} e_{p}(t-h)-\Delta A x(t) \\
& +\Delta A_{d} e_{p}(t-h)-\Delta A_{d} x(t-h)-\Delta B u(t), \\
\dot{e}_{s}(t)=- & \Delta A e_{p}(t)+A e_{s}(t)+B e_{f}(t)-\Delta B e_{u}(t) \\
& -\Delta A_{d} e_{p}(t-h)+A_{d} e_{s}(t-h) \\
& +\Delta A x(t)-H_{1} e_{y}(t)+\Delta B u(t)+\Delta A_{d} x(t-h), \\
\dot{e}_{f}(t)= & \dot{f}(t)-\dot{\hat{f}}(t)=-e_{f}(t)-H_{2} e_{y}+f(t)+\dot{f}(t) .
\end{aligned}
$$

The output estimation error $e_{y}(t)$ is given by

$$
\begin{aligned}
e_{y}(t)= & -\Delta C e_{p}(t)+C e_{s}(t)-\Delta C_{d} e_{p}(t-h) \\
& +C_{d} e_{s}(t-h)+\Delta C x(t)+\Delta C_{d} x(t-h) .
\end{aligned}
$$

The substitution of $e_{u}(t)$ in (8) implies

$$
\begin{aligned}
e_{u}(t)= & -K_{1} e_{p}(t)-K_{1} e_{s}(t)-K_{2} e_{p}(t-h) \\
& -K_{2} e_{s}(t-h)-e_{f}(t) .
\end{aligned}
$$

Equation (29) and (30) can be rewritten as

$$
\begin{aligned}
0 \dot{e}_{u}(t)= & K_{1} e_{p}(t)+K_{1} e_{s}(t)+K_{2} e_{p}(t-h) \\
& +K_{2} e_{s}(t-h)+e_{u}(t)+e_{f}(t), \\
0 \dot{e}_{y}(t)= & -\Delta C e_{p}(t)+C e_{s}(t)-e_{y}(t)-\Delta C_{d} e_{p}(t-h) \\
& +C_{d} e_{s}(t-h)+\Delta C_{d} x(t-h)+\Delta C x(t) .
\end{aligned}
$$

The combination of (28) and (31) leads to the following descriptor system:

$$
\begin{aligned}
E \dot{e}(t)= & (\widetilde{A}+\Delta \widetilde{A}) e(t)+\left(\widetilde{A}_{d}+\Delta \widetilde{A}_{d}\right) e(t-h) \\
& +(\widetilde{B}+\Delta \widetilde{B}) v(t),
\end{aligned}
$$

where $E, \widetilde{A}_{d}$, and $e(t)$ have been given above and the expressions of $\widetilde{A}, \Delta \widetilde{A}, \widetilde{B}, \Delta \widetilde{B}, \Delta \widetilde{A}_{d}$, and $v(t)$ are shown as follows:

$$
\widetilde{A}=\left[\begin{array}{ccccc}
A & 0 & 0 & B & 0 \\
0 & A & B & 0 & -H_{1} \\
0 & 0 & -I & 0 & -H_{2} \\
K_{1} & K_{1} & I & I & 0 \\
0 & C & 0 & 0 & -I
\end{array}\right],
$$$$
\Delta \widetilde{A}=\left[\begin{array}{ccccc}
\Delta A & 0 & 0 & \Delta B & 0 \\
-\Delta A & 0 & 0 & -\Delta B & 0 \\
0 & 0 & 0 & 0 & 0 \\
0 & 0 & 0 & 0 & 0 \\
-\Delta C & 0 & 0 & 0 & 0
\end{array}\right],
$$$$
\widetilde{B}=\left[\begin{array}{lllll}
0 & 0 & 0 & 0 & 0 \\
0 & 0 & 0 & 0 & 0 \\
0 & 0 & 0 & I & I \\
0 & 0 & 0 & 0 & 0 \\
0 & 0 & 0 & 0 & 0
\end{array}\right],
$$

$$
\Delta \widetilde{B}=\left[\begin{array}{ccccc}
-\Delta A & -\Delta A_{d} & -\Delta B & 0 & 0 \\
\Delta A & \Delta A_{d} & \Delta B & 0 & 0 \\
0 & 0 & 0 & 0 & 0 \\
0 & 0 & 0 & 0 & 0 \\
\Delta C & \Delta C_{d} & 0 & 0 & 0
\end{array}\right] \text {, }
$$

$$
\begin{aligned}
& \Delta \widetilde{A}_{d}=\left[\begin{array}{ccccc}
\Delta A_{d} & 0 & 0 & 0 & 0 \\
-\Delta A_{d} & 0 & 0 & 0 & 0 \\
0 & 0 & 0 & 0 & 0 \\
0 & 0 & 0 & 0 & 0 \\
-\Delta C_{d} & 0 & 0 & 0 & 0
\end{array}\right], \\
& v(t)=\left[x^{T}(t), x^{T}(t-h), u^{T}(t), f^{T}(t), \dot{f}^{T}(t)\right]^{T} .
\end{aligned}
$$

The condition ensuring the stability of the descriptor system (32) and the attenuation level $\gamma>0$ from the perturbation-like term $v(t)$ to the error dynamic $e(t)$ are provided in the following theorem.

Theorem 4. System (32) describing the different errors is stable and the gain from $v(t)$ to $e(t)$ is bounded by $\gamma>0$ if there exist some matrices $P_{1}>0, P_{2}>0$, and $P_{3}>0$ and matrices $Y_{2}, Y$ 
with appropriate dimensions and positive scalars $t_{1}, t_{2}, t_{3}$ such that the matrix inequality (34) holds.

$$
\left[\begin{array}{cccccc}
\widetilde{\Omega} & Y_{2} B & Y & Y_{2} M_{e a} & Y_{2} M_{e b} & Y_{2} M_{e a d} \\
* & -\gamma^{2} I+t_{2} N_{e b}^{T} N_{e b} & 0 & 0 & 0 & 0 \\
* & * & -\frac{P_{3}}{h} & 0 & 0 & 0 \\
* & * & * & -t_{1} I & 0 & 0 \\
* & * & * & * & -t_{2} I & 0 \\
* & * & * & * & * & -t_{3} I
\end{array}\right]<0,
$$

and the observer and controller gains are then computed by

$$
\begin{array}{ll}
K_{1}=\left(Y_{14}\right)^{-1} X_{41}, & K_{2}=\left(Y_{14}\right)^{-1} X_{42}, \\
H_{1}=\left(Y_{11}\right)^{-1} H_{21}, & H_{2}=\left(Y_{12}\right)^{-1} H_{32} .
\end{array}
$$

Proof. Let us consider the weighted $L_{2}$ constraint given by

$$
\int_{0}^{t} e^{T}(\sigma) Q e(\sigma) d \sigma<\gamma^{2} \int_{0}^{t} v^{T}(\sigma) v(\sigma) d \sigma
$$

where $\gamma$ is the attenuation level from the perturbation-like term $v(t)$ to the error $e(t)$ in (32) and $Q$ is a symmetric semipositive-definite weighted matrix. It is well known that the constraint is satisfied if there exists a Lyapunov-Krasovskii function such that

$$
\dot{V}(t)+e^{T}(t) Q e(t)-\gamma^{2} v^{T}(t) v(t)<0 .
$$

Choose Lyapunov-Krasovskii functional candidate as $V(t)=V_{1}(t)+V_{2}(t)+V_{3}(t)$ and $V_{1}(t), V_{2}(t), V_{3}(t)$ are defined in (22).

Adding $2 \Pi_{1}, 2 \Pi_{2}$, and $h \xi^{T}(t) Y P_{3}^{-1} Y^{T} \xi(t)$ and subtracting $\int_{t-h}^{t} \xi^{T}(t) Y P_{3}^{-1} Y^{T} \xi(t) d \omega$, respectively, in (37) yield (38) which is shown as follows:

$$
\begin{aligned}
\dot{V}(t) \leq & 2 e^{T}(t) E^{T} P_{1} \dot{e}(t)+h \dot{e}^{T}(t) E^{T} P_{3} E \dot{e}(t) \\
& -\int_{t-h}^{t} \dot{e}^{T}(\omega) E^{T} P_{3} E \dot{e}(\omega) d \omega+e^{T}(t) P_{2} e(t) \\
& -e^{T}(t-h) P_{2} e(t-h) \\
& +2 \xi^{T}(t) Y E\left[e(t)-e(t-h)-\int_{t-h}^{t} \dot{e}(\omega) d \omega\right] \\
& +2 \xi^{T}(t) Y_{2}\left[(\widetilde{A}+\Delta \widetilde{A}) e(t)+\left(\widetilde{A}_{d}+\Delta \widetilde{A}_{d}\right) e(t-h)\right. \\
& \quad+(\widetilde{B}+\Delta \widetilde{B}) v(t)-E \dot{e}(t)] \\
= & 2 e^{T}(t) P_{1} E \dot{e}(t)+h \dot{e}^{T}(t) E^{T} P_{3} E \dot{e}(t) \\
& -\int_{t-h}^{t} \dot{e}^{T}(\omega) E^{T} P_{3} E \dot{e}(\omega) d \omega+e^{T}(t) P_{2} e(t) \\
& -e^{T}(t-h) P_{2} e(t-h)
\end{aligned}
$$

$$
\begin{gathered}
+2 \xi^{T}(t) Y E\left[e(t)-e(t-h)-\int_{t-h}^{t} \dot{e}(\omega) d \omega\right] \\
+2 \xi^{T}(t) Y_{2}\left[\widetilde{A} e(t)+\widetilde{A}_{d} e(t-h)+\widetilde{B} v(t)-E \dot{e}(t)\right] \\
+\operatorname{sym}\left(\xi^{T}(t) Y_{2} M_{e a} F_{5}(t) N_{e a} e(t)\right. \\
+\xi^{T}(t) Y_{2} M_{e a d} F_{5}(t) N_{e a d} e(t-h) \\
\left.+\xi^{T}(t) Y_{2} M_{e b} F_{5}(t) N_{e b} v(t)\right) .
\end{gathered}
$$

By applying Lemma 1, (38) yields

$$
\begin{aligned}
\dot{V}(t) \leq & \xi^{T}(t) \widetilde{\Omega} \xi(t)+t_{1}^{-1} \xi^{T}(t) Y_{2} M_{e a} M_{e a}^{T} Y_{2}^{T} \xi(t) \\
& +t_{2}^{-1} \xi^{T}(t) Y_{2} M_{e a d} M_{e a d}^{T} Y_{2}^{T} \xi(t) \\
& +t_{3}^{-1} \xi^{T}(t) Y_{2} M_{e b} M_{e b}^{T} Y_{2}^{T} \xi(t) \\
& +2 \xi^{T}(t) Y_{2} \widetilde{B} v(t)+t_{2} v^{T}(t) N_{e b}^{T} N_{e b} v(t),
\end{aligned}
$$

where the $\widetilde{\Omega}$ is expressed as follows:

$$
\begin{aligned}
\widetilde{\Omega}= & W_{p} Q W_{p}^{T}+t_{1} W_{p} N_{e a}^{T} N_{e a} W_{p}^{T} \\
& +t_{3} W_{p h} N_{e a d}^{T} N_{e a d} W_{p h}^{T}+W_{p} P_{2} W_{p}^{T} \\
& +h W_{p 1} E^{T} P_{3} E W_{p 1}^{T}-W_{p h} P_{2} W_{p h}^{T} \\
& +\operatorname{sym}\left(W_{p} P_{1} W_{p 1}^{T}+Y E W_{P}^{T}+Y E W_{p h}^{T}\right. \\
& +\left(Y_{2} A_{0}-H C_{1}-X C_{2}\right) W_{p}^{T} \\
& \left.+\left(Y_{2} A_{d 0}-K C_{3}\right) W_{p h}^{T}\right) .
\end{aligned}
$$

$W_{p}, W_{p 1}, W_{p h}, A_{0}, A_{d 0}, \widetilde{Y}_{2}, X, K, H, C_{1}, C_{2}$, and $C_{3}$ are defined by Theorem 2 and $M_{e a}, M_{e b}, M_{e a d}, N_{e a}, N_{e b}$, and $N_{e a d}$ are shown as follows:

$$
\begin{gathered}
M_{e a}=\left[\begin{array}{ccccc}
M_{a} & 0 & M_{b} & 0 & 0 \\
0 & M_{a} & 0 & M_{b} & 0 \\
0 & 0 & 0 & 0 & 0 \\
0 & 0 & 0 & 0 & 0 \\
0 & 0 & 0 & 0 & 0
\end{array}\right], \\
N_{e a}=\left[\begin{array}{ccccc}
N_{a} & 0 & 0 & 0 & 0 \\
-N_{a} & 0 & 0 & 0 & 0 \\
0 & 0 & 0 & N_{b} & 0 \\
0 & 0 & 0 & -N_{b} & 0 \\
-N_{c} & 0 & 0 & 0 & M_{c}
\end{array}\right], \\
M_{e a d}=\left[\begin{array}{ccccc}
M_{a d} & 0 & 0 & 0 & 0 \\
-M_{a d} & 0 & 0 & 0 & 0 \\
0 & 0 & 0 & 0 & 0 \\
0 & 0 & 0 & 0 & 0 \\
0 & M_{c d} & 0 & 0 & 0
\end{array}\right],
\end{gathered}
$$




$$
\begin{aligned}
& N_{e a d}=\left[\begin{array}{ccccc}
N_{a d} & 0 & 0 & 0 & 0 \\
-N_{a d} & 0 & 0 & 0 & 0 \\
0 & 0 & 0 & 0 & 0 \\
0 & 0 & 0 & 0 & 0 \\
0 & 0 & 0 & 0 & 0
\end{array}\right], \\
& M_{e b}=\left[\begin{array}{ccccc}
-M_{a} & M_{b} & -M_{a d} & 0 & 0 \\
M_{a} & -M_{b} & M_{a d} & 0 & 0 \\
0 & 0 & 0 & 0 & 0 \\
0 & 0 & 0 & 0 & 0 \\
0 & 0 & 0 & M_{c} & M_{c d}
\end{array}\right], \\
& N_{e b}=\left[\begin{array}{ccccc}
N_{a} & 0 & 0 & 0 & 0 \\
0 & 0 & -N_{b} & 0 & 0 \\
0 & N_{a d} & 0 & 0 & 0 \\
N_{c} & 0 & 0 & 0 & 0 \\
0 & N_{c d} & 0 & 0 & 0
\end{array}\right] .
\end{aligned}
$$

Following the similar steps of previous proofs and applying Schur complement on (39), the sufficient LMI conditions proposed in Theorem 4 follow.

Remark 5. It should be mentioned that our study to this paper is mainly motivated by the work of [17]. There are three differences between the work given in [17] and ours. First, the system in [17] are Takagi-sugeno fuzzy models and in our study, the linear system is investigated; however, the results of the linear system theories can be applied for the design of Takagi-sugeno fuzzy models. Second, the Takagi-sugeno models of the work [17] taken are combinations of linear time invariant systems. Our study is the extend of linear systems to linear time-delay systems, but it is not taken into account in [17]. Third, problems of the stability and the $H_{\infty}$ control for delayed systems use the Lyapunov-Krasovskii approach other than the Lyapunov-Razumikhin approach and the results of using Lyapunov-Krasovskii approach are usually less conservative than those using Lyapunov-Razumikhin approach [25].

\section{Numerical Example}

In this section, we will provide a numerical example to demonstrate the effectiveness of the design methods proposed in the previous section. This example is taken from [23] and the faulty system is defined by

$$
\begin{gathered}
A=\left[\begin{array}{cc}
-1.4274 & 0.0757 \\
-1.4189 & -0.9442
\end{array}\right], \quad B=\left[\begin{array}{c}
0 \\
0.3
\end{array}\right], \\
A_{d}=\left[\begin{array}{cc}
0.25 & 0 \\
0 & 0.25
\end{array}\right], \quad C=\left[\begin{array}{cc}
0.1 & 0 \\
0 & 0.1
\end{array}\right], \\
C_{d}=\left[\begin{array}{cc}
0.1 & 0 \\
0 & 0.1
\end{array}\right] .
\end{gathered}
$$

We assume that the time delay is given by $h=0.1$. Letting the nominal input signal $u(t)=\sin (t)$ and the constant fault $f=1$ affecting the system behavior at $30 \mathrm{~s} \leq t \leq 100 \mathrm{~s}$, one

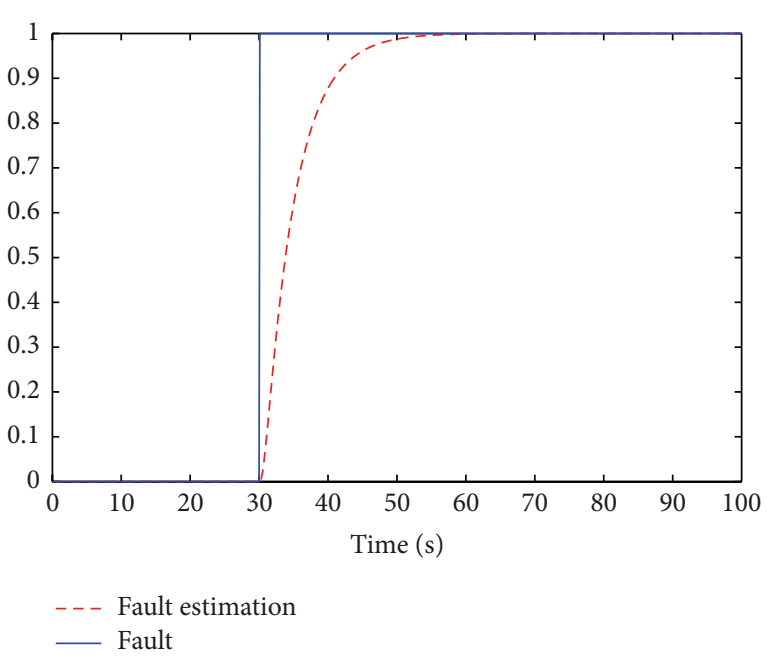

FIGURE 2: Fault and its estimation.

can obtain the following solution by solving the conditions in the Theorem 2:

$$
\begin{aligned}
& H_{1}=\left[\begin{array}{cc}
0.7233 & 2.3038 \\
-9.2496 & 5.2505
\end{array}\right], \\
& H_{2}=\left[\begin{array}{ll}
-1.8912 & 5.6556
\end{array}\right], \\
& K_{1}=\left[\begin{array}{ll}
-0.1582 & 0.0076
\end{array}\right], \\
& K_{2}=\left[\begin{array}{ll}
0.2101 & 0.7059
\end{array}\right] .
\end{aligned}
$$

The simulation results are shown in Figures 2, 3, 4, and 5. In Figure 2, the real fault and its estimate are depicted. Figures 3 and 4 compare the state variables of the reference model, the observer, and the faulty system with FTC. Figure 5 shows the comparison of the nominal control input and FTC signal.

In the next, we will consider the time-varying fault with system uncertainties; the uncertainties are defined by

$$
\begin{aligned}
& M_{a d}=M_{b}=M_{a}=\operatorname{diag}\{0.1,0.1\}, \\
& M_{c}=M_{c d}=\operatorname{diag}\{0.1,0.1\}, \\
& N_{a}=N_{a d}=N_{c}=N_{c d}=\operatorname{diag}\{0.01,0.01\}, \\
& N_{b}=[0.01,0.01]^{T} .
\end{aligned}
$$

The system uncertainties are given by $F(t)=0.5 \sin (t)$. The definition of the time delay $h$ and the nominal input signal $u(t)$ is given by $h=0.1$ and $u(t)=\sin (t)$. The timevarying fault $f=\sin (0.5 * t-8) * \cos (t)$ affects the system behavior at $30 \mathrm{~s} \leq t \leq 100 \mathrm{~s}$.

When choosing the free weighting matrix $Y_{2}=$ $\left[\begin{array}{lll}\widetilde{Y}_{2}^{T} & 0 & \widetilde{Y}_{2}^{T}\end{array}\right]^{T}$ and solving the conditions in Theorem 4 with 


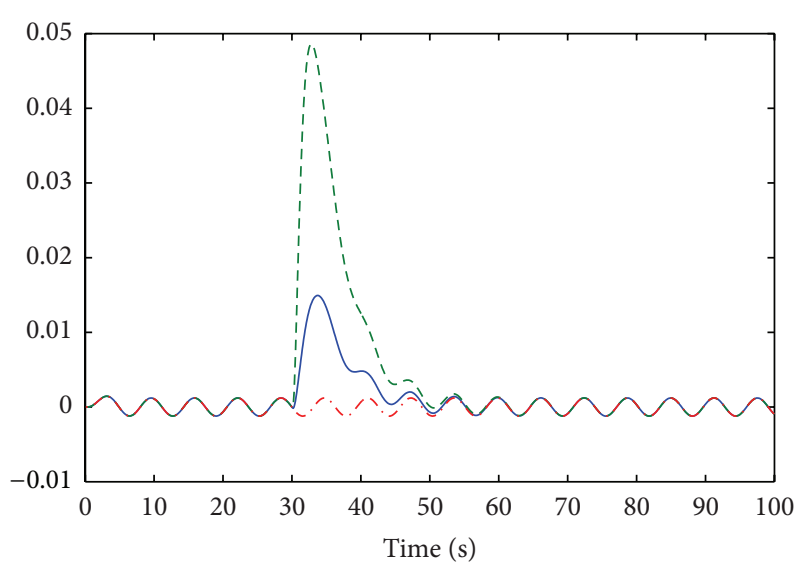

_ State of faulty system _..- State of reference system

_. - State of observer

Figure 3: Comparison of reference mode state $x_{1}$, faulty system state $x_{f 1}$, and the observer state $\widehat{x}_{f 1}$.

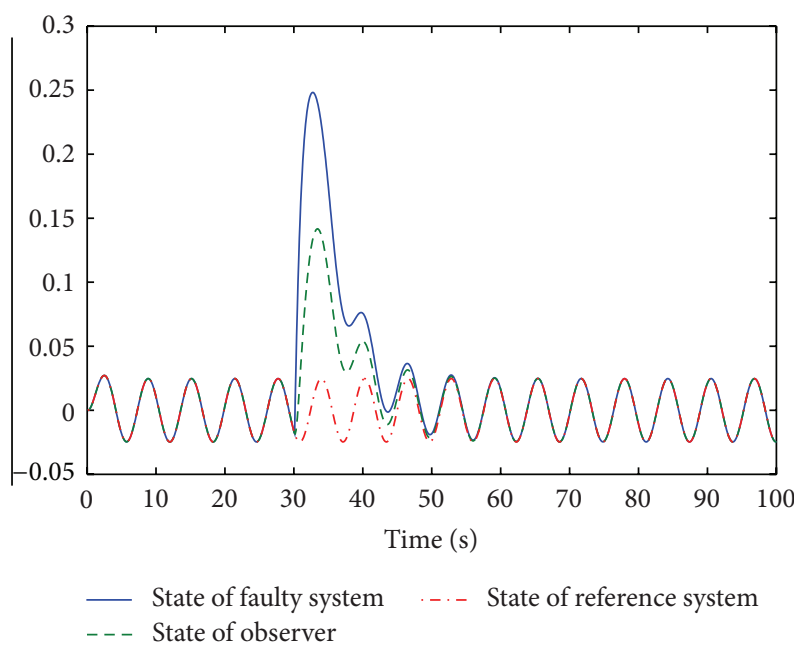

Figure 4: Comparison of reference mode state $x_{2}$, faulty system state $x_{f 2}$, and the observer state $\hat{x}_{f 2}$.

$Q=\operatorname{diag}\left\{I_{2}, I_{2}, I_{1}, I_{1}, I_{2}\right\}$, one can obtain the attenuation value $\gamma=0.346928$ with

$$
\begin{aligned}
H_{1} & =\left[\begin{array}{cc}
0.9485 & 2.1521 \\
3.8575 & 109.0013
\end{array}\right], \\
H_{2} & =\left[\begin{array}{ll}
0.0278 & 2.5129
\end{array}\right] \times 10^{3}, \\
K_{1} & =\left[\begin{array}{ll}
28.9781 & -2.7970
\end{array}\right], \\
K_{2} & =\left[\begin{array}{ll}
4.1592 & 3.4426
\end{array}\right] .
\end{aligned}
$$

In order to show the effective influence of the FTC on the system trajectory, we make the comparison between the systems with and without FTC when faults occur. The simulation results are shown in Figures 6, 7, 8, 9, 10, and 11. Figure 6 illustrates the simulation result of fault estimation. Figures 7 and 8 show the state variables of the reference

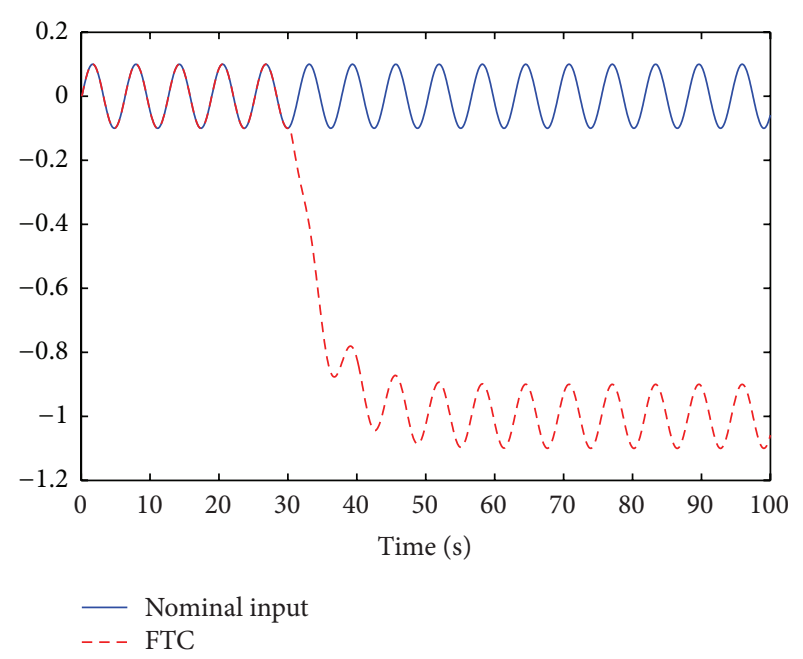

FIgURE 5: Nominal input $u(t)$ and FTC $u_{f}(t)$.

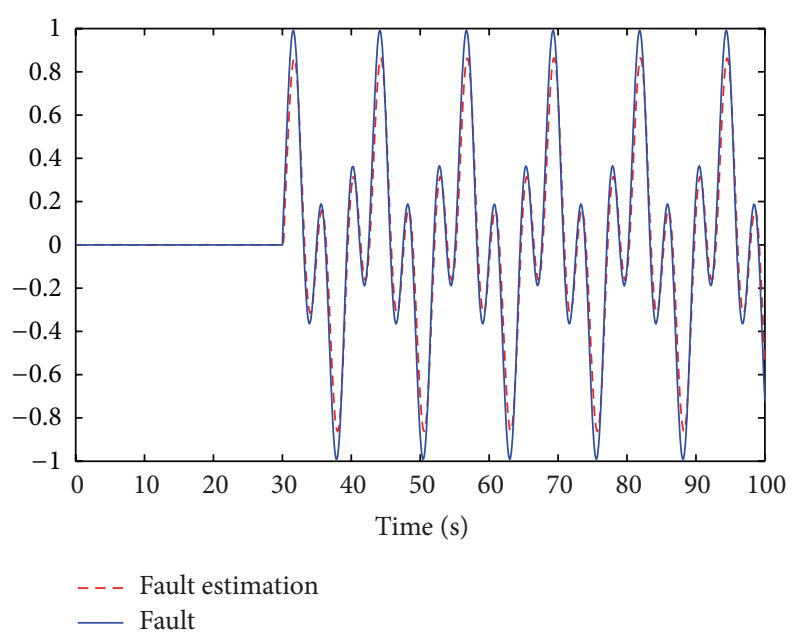

Figure 6: Fault and its estimation.

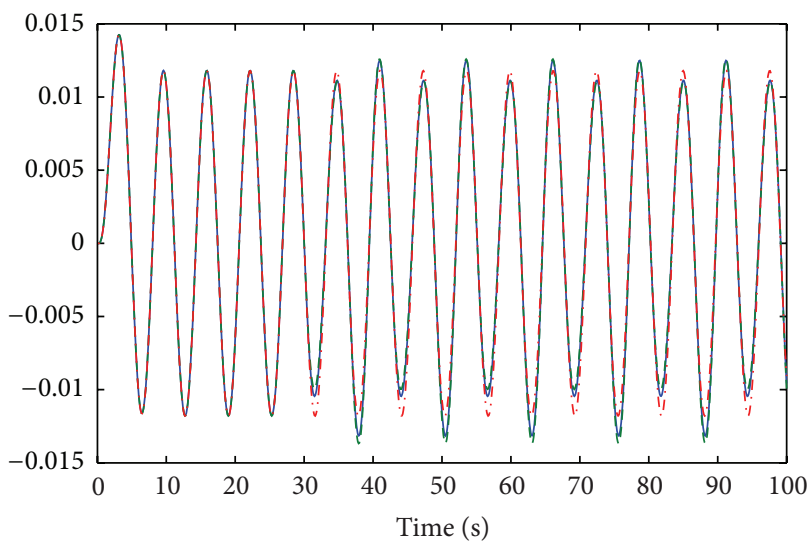

_ State of faulty system _... State of reference system
_ - State of observer

FIGURE 7: Comparison of reference mode state $x_{1}$, faulty system state $x_{f 1}$, and the observer state $\widehat{x}_{f 1}$ with FTC. 


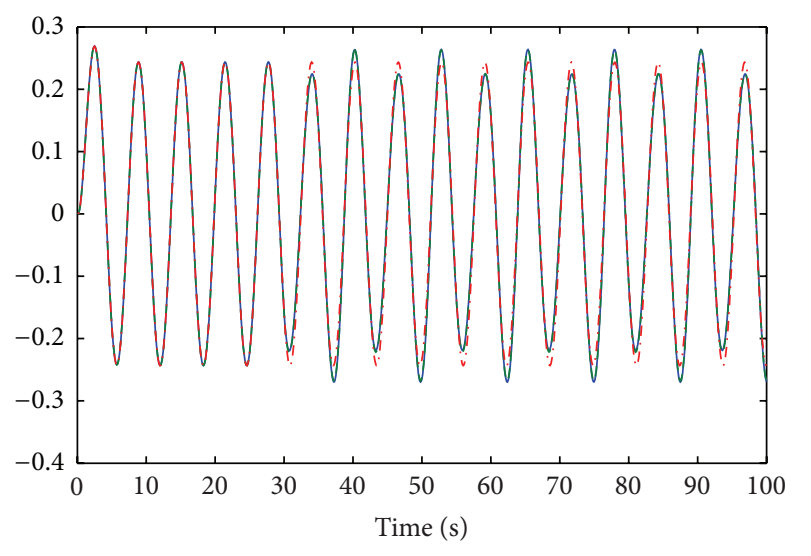

__ State of faulty system _..- State of reference system - - - State of observer

FIGURE 8: Comparison of reference mode state $x_{2}$, faulty system state $x_{f 2}$, and the observer state $\widehat{x}_{f 2}$ with FTC.

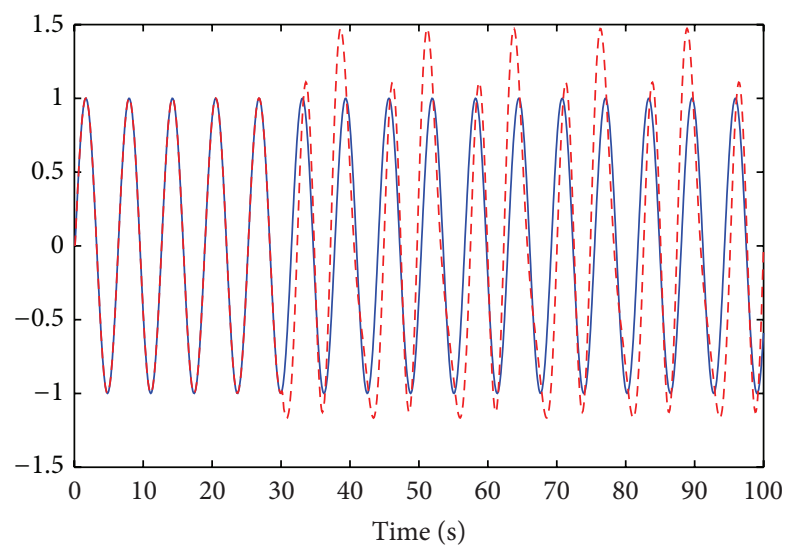

- Nominal input

- - FTC

FIGURE 9: Nominal input $u(t)$ and FTC $u_{f}(t)$.

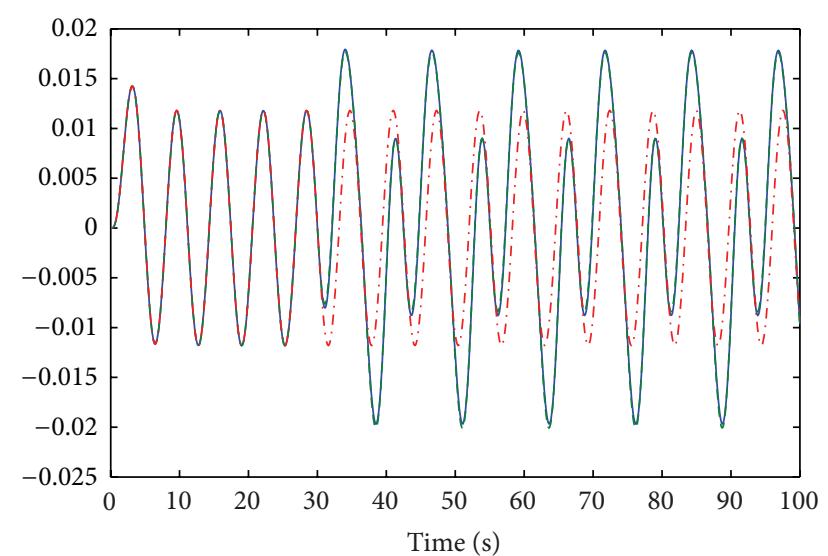

_ State of faulty system _... State of reference system

- - State of observer

FIgURE 10: Comparison of reference mode state $x_{1}$, faulty system state $x_{f 1}$, and the observer state $\hat{x}_{f 1}$ without FTC.

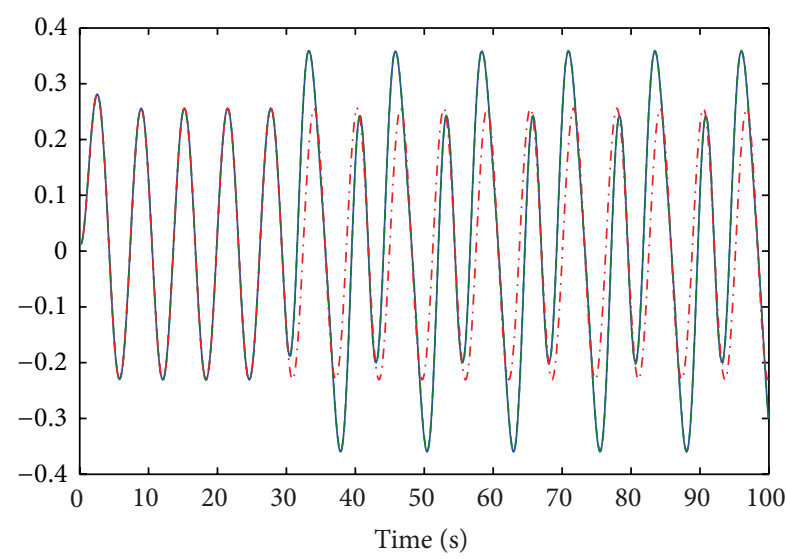

_ State of faulty system _..- State of reference system
_ - State of observer

FIGURE 11: Comparison of reference mode state $x_{2}$, faulty system state $x_{f 2}$, and the observer state $\widehat{x}_{f 2}$ without FTC.

model, the observer, and the faulty system with FTC. The comparison of the nominal control and FTC signal is shown in Figure 9. Figures 10 and 11 show the state variables of the reference model, the observer, and the faulty system without FTC.

Two different cases are considered including constant fault without uncertainties and time-varying fault with uncertainties. From the above simulation results, one can see that the synthesized observers and FTC controllers showed their effectiveness, since the fault is estimated (Figures 2 and 6) and the state variables of the faulty system with FTC are closed to reference model (Figures 3, 4, 7, and 8), and the tracking between the faulty system and the reference model is ensured. On the other hand, if FTC is not employed in the faulty system (Figures 10 and 11), the state trajectories of faulty system deviate from the state trajectories of reference model and the tracking is not achieved.

\section{Conclusion}

In this paper, the problem of active FTC design for linear time-delay systems with and without uncertainties is treated. The aim of the FTC law and observer design is to ensure the trajectory tracking of faulty system. By considering the descriptor redundancy of closed-loop systems and using Lyapunov-Krasovskii functional approach, the proposed FTC scheme has been easily formulated in LMI terms. Two kinds of faults have been considered. The first one deals with the constant faults without uncertainties as a special case, and the other deals with the time-varying faults with system uncertainties. Finally, one example has been considered to illustrate the efficiency of the proposed scheme in both cases. In addition, it is interesting to develop the FTC control law by taking into account modeling multiplicative faults and some external perturbations and considering how to deal with sensor faults and how to apply this scheme to T-S models. 


\section{Conflict of Interests}

The authors declare that there is no conflict of interests regarding the publication of this paper.

\section{Acknowledgments}

The authors would like to thank the reviewers for their constructive comments and suggestions which have helped to improve the presentation of the paper. This work was supported by the National Natural Science Foundation of China (no. 61300115).

\section{References}

[1] J. Chen and R. J. Patton, Robust Model-Based Fault Diagnosis for Dynamic Systems, Kluwer Academic Publishers, Boston, Mass, USA, 1999.

[2] M. Blanke, M. Kinnaert, and J. Lunze, Diagnosis and FaultTolerant Control, Springer, Berlin, Germany, 2006.

[3] H. Niemann and J. Stoustrup, "Passive fault tolerant control of a double inverted pendulum - a case study," Control Engineering Practice, vol. 13, no. 8, pp. 1047-1059, 2005.

[4] H. Wang and S. Daley, "Actuator fault diagnosis: an adaptive observer-based technique," IEEE Transactions on Automatic Control, vol. 41, no. 7, pp. 1073-1078, 1996.

[5] B. Jiang, M. Staroswiecki, and V. Cocquempot, "Fault identification for a class of time-delay systems," in Proceedings of the American Control Conference, pp. 2239-2244, Anchorage, Alaska, USA, May 2002.

[6] C. Edwards, S. K. Spurgeon, and R. J. Patton, "Sliding mode observers for fault detection and isolation," Automatica, vol. 36, no. 4, pp. 541-553, 2000.

[7] N. E. Wu, Y. Zhang, and K. Zhou, "Detection, estimation, and accommodation of loss of control effectiveness," International Journal of Adaptive Control and Signal Processing, vol. 14, no. 7, pp. 775-795, 2000.

[8] B. Marx, D. Koenig, and D. Georges, "Robust fault-tolerant control for descriptor systems," IEEE Transactions on Automatic Control, vol. 49, no. 10, pp. 1869-1875, 2004.

[9] M. Wang, B. Chen, and S. Tong, "Adaptive fuzzy tracking control for strict-feedback nonlinear systems with unknown time delays," International Journal of Innovative Computing, Information and Control, vol. 4, no. 4, pp. 829-837, 2008.

[10] W. Chen and M. Saif, "An iterative learning observer for fault detection and accommodation in nonlinear time-delay systems," International Journal of Robust and Nonlinear Control, vol. 16, no. 1, pp. 1-19, 2006.

[11] W. Chen and M. Saif, "Fault detection and accommodation in nonlinear time-dealy systems," in Proceedings of the American Control Conference, pp. 4255-4260, June 2003.

[12] B. Jiang, M. Staroswiecki, and V. Cocquempot, " $H_{\infty}$ fault detection filter design for linear discretetime systems with multiple time delays," International Journal of Systems Science, vol. 34, no. 5, pp. 365-373, 2003.

[13] D. Du, B. Jiang, and P. Shi, "Sensor fault estimation and compensation for time-delay switched systems," International Journal of Systems Science, vol. 43, no. 4, pp. 629-640, 2012.

[14] C. Jiang, D. H. Zhou, and F. Gao, "Robust fault detection and isolation for uncertain linear retarded systems," Asian Journal of Control, vol. 8, no. 2, pp. 141-152, 2006.
[15] D. Zhang, L. Yu, and W.-A. Zhang, "Delay-dependent fault detection for switched linear systems with time-varying delaysthe average dwell time approach," Signal Processing, vol. 91, no. 4, pp. 832-840, 2011.

[16] B. Jiang, K. Zhang, and P. Shi, "Less conservative criteria for fault accommodation of time-varying delay systems using adaptive fault diagnosis observer," International Journal of Adaptive Control and Signal Processing, vol. 24, no. 4, pp. 322-334, 2010.

[17] T. Bouarar, B. Marx, D. Maquin, and J. Ragot, "Fault-tolerant control design for uncertain Takagi-Sugeno systems by trajectory tracking: a descriptor approach," IET Control Theory \& Applications, vol. 7, no. 14, pp. 1793-1805, 2013.

[18] D. Ichalal, B. Marx, J. Ragot, and D. Maquin, "Observer based actuator fault tolerant control for nonlinear TakagiSugeno systems: an LMI approach," in Proceedings of the 18th Mediterranean Conference on Control and Automation (MED '10), pp. 1278-1283, Marrakech, Morocco, June 2010.

[19] T. Bouarar, B. Marx, D. Maquin, and J. Ragot, "Trajectory tracking fault tolerant controller design for Takagi-Sugeno systems subject to actuator faults," in Proceedings of the International Conference on Communications, Computing and Control Applications (CCCA '11), pp. 1-6, Hammamet, Tunisia, March 2011.

[20] K. Guelton, T. Bouarar, and N. Manamanni, "Robust dynamic output feedback fuzzy Lyapunov stabilization of Takagi-Sugeno systems-a descriptor redundancy approach," Fuzzy Sets and Systems, vol. 160, no. 19, pp. 2796-2811, 2009.

[21] T. Bouarar, K. Guelton, and N. Manamanni, "Static output feedback controller design for Takagi-Sugeno systems-a fuzzy Lyapunov LMI approach," in Proceedings of the 48th IEEE Conference on Decision and Control Held Jointly with 28th Chinese Control Conference (CDC/CCC '09), pp. 4150-4155, IEEE, Shanghai , China, December 2009.

[22] T. Bouarar, K. Guelton, and N. Manamanni, "Robust fuzzy Lyapunov stabilization for uncertain and disturbed TakagiSugeno descriptors," ISA Transactions, vol. 49, no. 4, pp. 447$461,2010$.

[23] Y. Zhao, J. Wu, and P. Shi, "Ho control of non-linear dynamic systems: a new fuzzy delay partitioning approach," IET Control Theory \& Applications, vol. 3, no. 7, pp. 917-928, 2009.

[24] X. F. Jiang and Q.-L. Han, "Robust $H_{\infty}$ control for uncertain Takagi-Sugeno fuzzy systems with interval time-varying delay," IEEE Transactions on Fuzzy Systems, vol. 15, no. 2, pp. 321-331, 2007.

[25] G. J. Zhang, C. S. Han, and L. G. Wu, "Admissibility and $H_{\infty}$ performance analysis of T-S fuzzy descriptor systems with timedelay," in Proceedings of the 2nd International Conference on Intelligent Control and Information Processing (ICICIP '11), pp. 77-81, July 2011. 


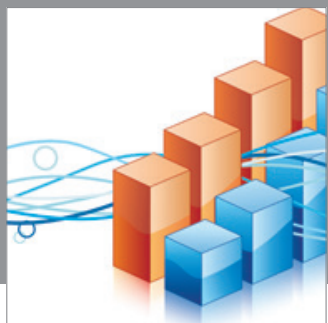

Advances in

Operations Research

mansans

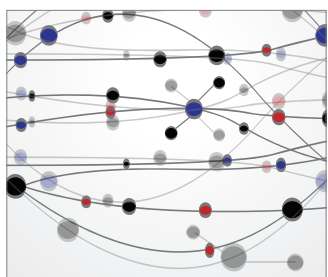

The Scientific World Journal
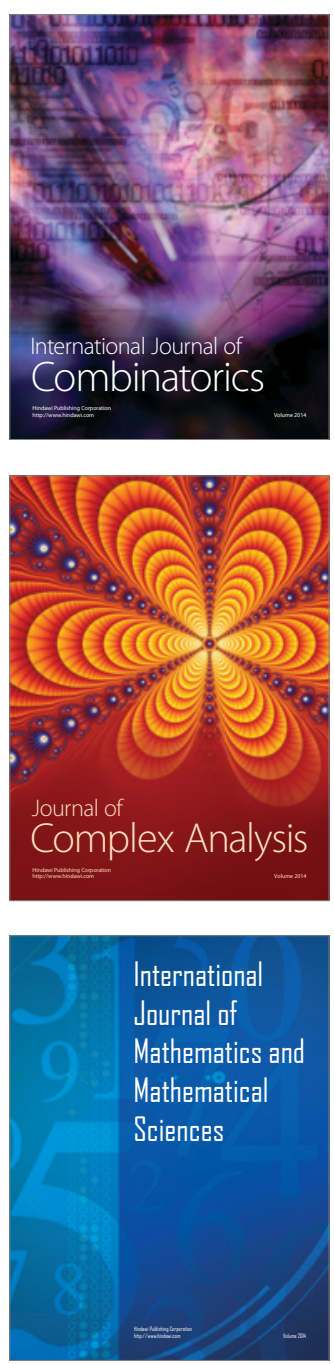
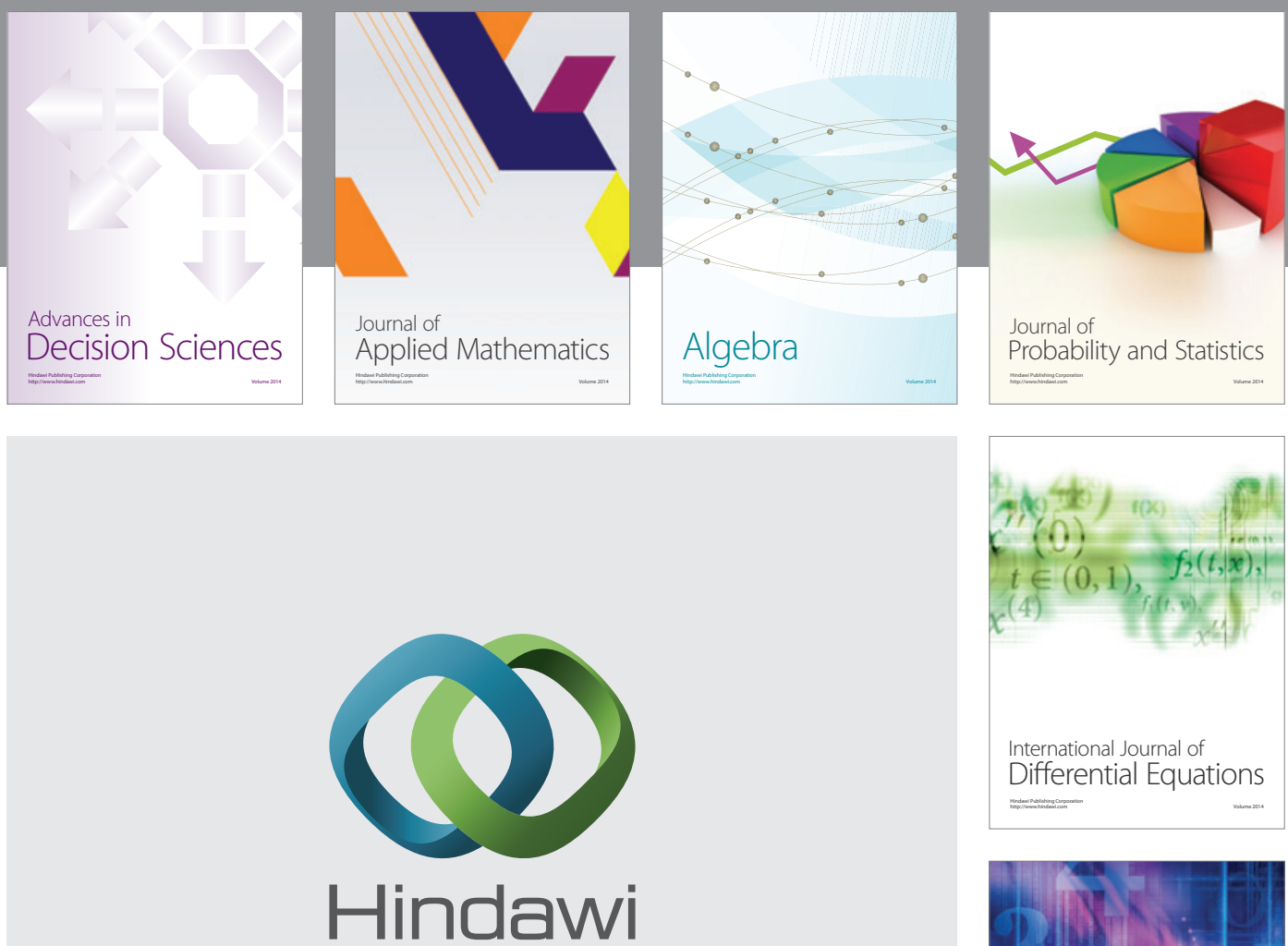

Submit your manuscripts at http://www.hindawi.com
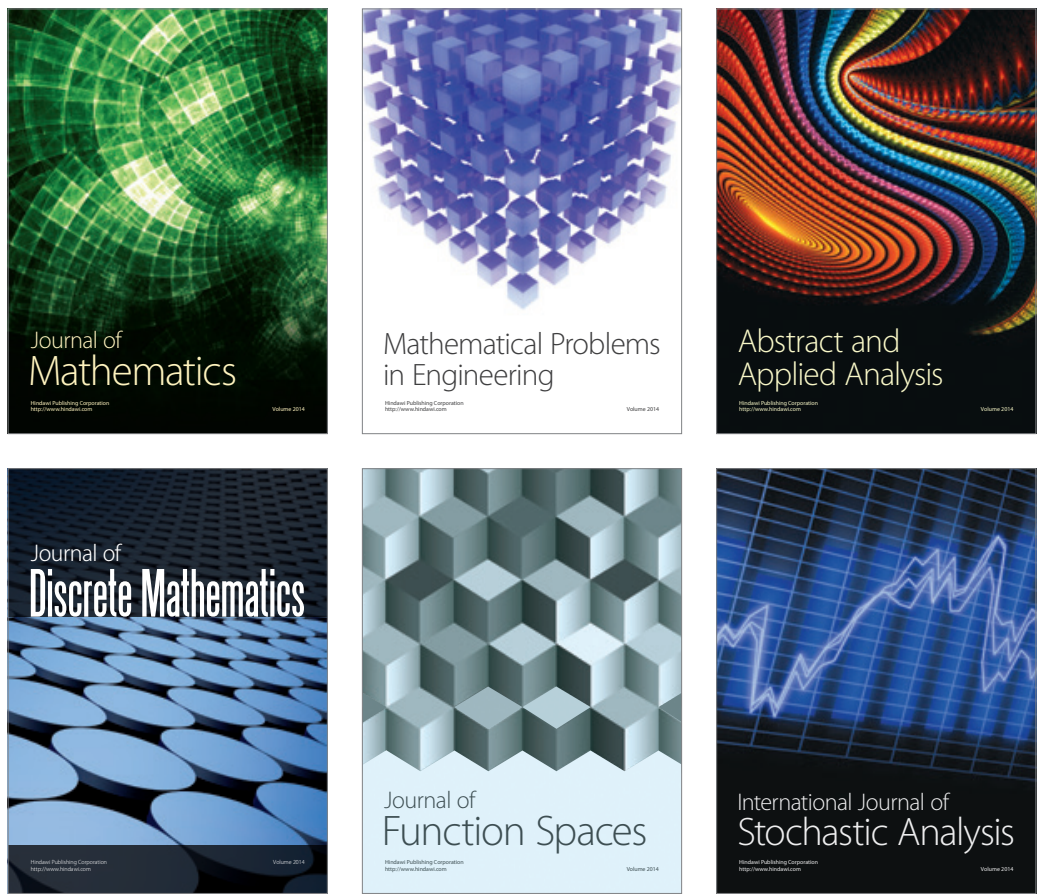

Journal of

Function Spaces

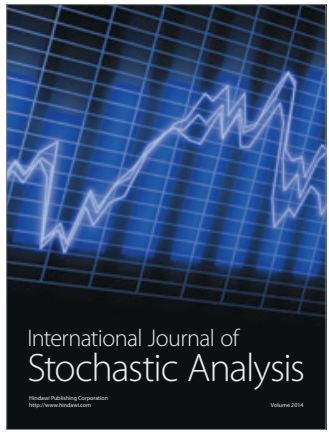

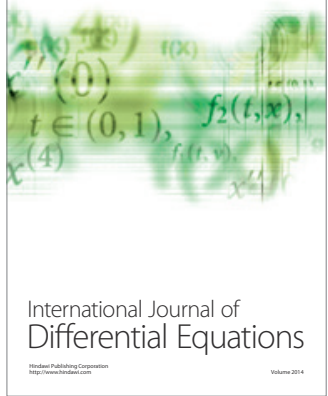
